\title{
Modulation of ionotropic glutamate receptors and acid-sensing ion channels by nitric oxide
}

\author{
John Q. Wang ${ }^{1,2}{ }^{*}$, Xiang-Ping Chu ${ }^{1}$, Ming-Lei Guo ${ }^{1}$, Dao-Zhong Jin ${ }^{1}$, Bing Xue ${ }^{1}$, Thomas J. Berry ${ }^{2}$, \\ Eugene E. Fibuch ${ }^{2}$ and Li-Min Mao'
}

${ }^{1}$ Department of Basic Medical Science, School of Medicine, University of Missouri-Kansas City, Kansas City, MO, USA

${ }^{2}$ Department of Anesthesiology, School of Medicine, University of Missouri-Kansas City, Kansas City, MO, USA

\section{Edited by:}

Mike Althaus, Justus-Liebig

University of Giessen, Germany

Reviewed by:

Francisco Fernandez De-Miguel, Universidad Nacional Autonoma de Mexico, Mexico

Teresa Giraldez, University Hospital

NS Candelaria, Spain

\section{*Correspondence:}

John Q. Wang, Department of Basic Medical Science, School of Medicine, University of Missouri-Kansas City, 2411 Holmes Street, Kansas City, MO 64108, USA.

e-mail:wangjq@umkc.edu
Ionotropic glutamate receptors (iGluR) are ligand-gated ion channels and are densely expressed in broad areas of mammalian brains. Like iGluRs, acid-sensing ion channels (ASIC) are ligand $\left(\mathrm{H}^{+}\right)$-gated channels and are enriched in brain cells and peripheral sensory neurons. Both ion channels are enriched at excitatory synaptic sites, functionally coupled to each other, and subject to the modulation by a variety of signaling molecules. Central among them is a gasotransmitter, nitric oxide (NO). Available data show that NO activity-dependently modulates iGluRs and ASICs via either a direct or an indirect pathway. The former involves a NO-based and cGMP-independent post-translational modification (Snitrosylation) of extracellular cysteine residues in channel subunits or channel-interacting proteins. The latter is achieved by NO activation of soluble guanylyl cyclase, which in turn triggers an intracellular cGMP-sensitive cascade to indirectly modulate iGluRs and ASICs. The NO modification is usually dynamic and reversible. Modified channels undergo significant, interrelated changes in biochemistry and electrophysiology. Since NO synthesis is enhanced in various neurological disorders, the NO modulation of iGluRs and ASICs is believed to be directly linked to the pathogenesis of these disorders. This review summarizes the direct and indirect modifications of iGluRs and ASICs by NO and analyzes the role of the NO-iGluR and NO-ASIC coupling in cell signaling and in the pathogenesis of certain related neurological diseases.

Keywords: NMDA, AMPA, ASIC, nitrosylation, cGMP, NO, NOS, gasotransmitter

\section{INTRODUCTION}

Ionotropic glutamate receptors (iGluR) are ligand-gated cation ion channels. They are classified into $N$-methyl-D-aspartate receptors (NMDAR), $\alpha$-amino-3-hydroxy-5-methylisoxazole-4propionic acid receptors (AMPAR), and kainate receptors (Dingledine et al., 1999). These receptors are densely distributed in broad regions of the mammalian central nervous system (CNS). Functional iGluRs are assembled by distinct subunits. Activation of iGluRs by an endogenous ligand, i.e., neurotransmitter glutamate, opens the channel for the $\mathrm{Na}^{+}$flow into cells, resulting in depolarization. NMDARs and GluR2-lacking AMPARs also allow $\mathrm{Ca}^{2+}$ influx for triggering multifunctional $\mathrm{Ca}^{2+}$-associated signaling pathways. Compared to NMDARs, AMPARs open and close more quickly, and thus mediate most of the fast excitatory synaptic transmission in the CNS. As a major group of excitatory receptors, iGluRs are critical for normal operations of cellular and synaptic activity and plasticity. Malfunction of these ion channels is thus frequently linked to the pathogenesis of a wide range of neurological disorders (Dingledine et al., 1999).

Neuronal cells are sensitive to changing extracellular proton (acid) concentrations. In sensory neurons, an acid-triggered inward ion current was first observed in 1980 (Krishtal and Pidoplichko, 1980). This current was then found to be mediated through the membrane-bound acid-sensing ion channel (ASIC;
Waldmann et al., 1997b). ASICs are a distinct family of proton $\left(\mathrm{H}^{+}\right)$-gated, voltage-independent, and cation $\left(\mathrm{Na}^{+}\right)$-selective channels. They are broadly expressed in peripheral sensory neurons and CNS neurons (Waldmann et al., 1997a,b; Alvarez de la Rosa et al., 2003). More specifically, among all ASIC subunits so far identified, ASIC1a, 2a, and 2b are expressed in CNS neurons at a high level, while all other ASICs are expressed in peripheral sensory neurons. In addition, ASICs are seen in non-neuronal cells, such as vascular smooth muscles and bone (Jahr et al., 2005; Grifoni et al., 2008). ASICs are important for maintaining cell homeostasis in response to normal changes in $\mathrm{pH}$ signals and are involved in discrete disorders where a dramatic drop in extracellular $\mathrm{pH}$ values (acidosis) occurs, such as seen in inflammation, ischemic stroke, traumatic brain injury, and acid nociception (Wemmie et al., 2006; Chu et al., 2011).

Like many other ion channels, iGluRs and ASICs are subject to the modulation by various extracellular and intracellular signals. This modulation is essential for normal channel operations and for adjusting the efficacy and strength of channels to properly respond to a given stimulus. Among important modulators is nitric oxide (NO), a short-lived and plasma membrane diffusible gasotransmitter. Increasing evidence shows that NO can modulate iGluRs and ASICs through a direct (cGMP-independent) or an indirect (cGMP-dependent) pathway (Ahern et al., 2002). 
The direct pathway involves a NO-based post-translational modification of iGluR and ASIC proteins, i.e., S-nitrosylation. This biochemical reaction occurs when a nitrosyl group is added to the thiol side-chain of cysteine residues to form $S$-nitrosothiols, leading to changes in tertiary structure and function of modified iGluRs and ASICs (Sen and Snyder, 2010). The indirect pathway starts with NO stimulation of soluble guanylyl cyclase, leading to an increase in the second messenger cGMP production and activation of cGMP-dependent downstream protein kinases (Bredt and Snyder, 1989). This review will discuss current progress in understanding the modulation of iGluRs and ASICs by NO. We reviewed these two types of channels together because (1) they are co-localized in the confined postsynaptic density microdomain (Zha et al., 2006), (2) they are functionally coupled to each other to contribute to neurological disorders involving excessive glutamate release and acidosis (Gao et al., 2005), and (3) they show some common properties in the regulation by NO.

\section{MODULATION OF iGluRs BY NO NMDARs}

The NMDAR is a unique ion channel that is both ligand-gated and voltage-dependent. Co-activation of NMDARs by the endogenous transmitters (glutamate and glycine) and the release of voltagedependent $\mathrm{Mg}^{2+}$ block trigger the opening of ion channels, allowing the inward flow of $\mathrm{Na}^{+}$and small amounts of $\mathrm{Ca}^{2+}$ into cells (Dingledine et al., 1999). The $\mathrm{Ca}^{2+}$ influx is critical for a variety of NMDAR signaling and function. Functional NMDARs are an assembly of obligatory NR1 and the modulatory NR2 subunits. Among all of the modulatory subunits (A-D), NR2A and NR2B are predominant NMDAR components and are mostly enriched in neurons (Stephenson, 2001).

NMDARs are closely linked to the NO system. Activation of NMDARs consistently increases activity of neuronal nitric oxide synthase (nNOS) in the cytoplasm of neurons. Active nNOS then catalyzes the production of endogenous NO from L-arginine, leading to an increased release of NO from neurons (Garthwaite et al., 1988; Shibuki and Okada, 1991; Bredt and Snyder, 1992). A number of studies in vitro and in vivo have demonstrated a significant role of $\mathrm{NO}$ in the regulation of normal NMDAR function. In cultured neurons, the NO-producing agent 3-morpholino-sydnonimine (SIN-1) inhibited NMDA-induced currents and associated increases in $\mathrm{Ca}^{2+}$ influx (Manzoni et al., 1992). Similar results were observed with 1-nitrosopyrrolidine (an NO-containing agent) and $\mathrm{NaNO}_{2}$ (a released form of NO), while NO scavenging by hemoglobin reversed the effect of these agents (Manzoni et al., 1992). Endogenous NOS activity in cultured striatal neurons is also closely linked to NMDAR activity. The natural substrate of NOS, L-arginine, diminished NMDAR activity, which was reversed by the NOS inhibitor L-nitroarginine (Manzoni and Bockaer, 1993). In channel recordings from rat cortical neurons or Xenopus oocytes, the NO-generating agent $S$-nitrosocysteine (SNOC) decreased NMDAR channel opening (Lei et al., 1992; Lipton et al., 1998) or NMDA-evoked currents (Omerovic et al., 1995; Choi et al., 2000). Similar to the in vitro results, an in vivo microdialysis study showed that the NO donor $S$-nitroso- $N$-acetylpenicillamine (SNAP) suppressed the NMDAstimulated NO release in the rat striatum (Kendrick et al., 1996).
Together, in vitro and in vivo data support an inhibitory modulation of NMDARs by NO under normal conditions. Given that NMDAR stimulation increases $\mathrm{NO}$ synthesis, $\mathrm{NO}$ and NO-related species serve as key regulators in a use-dependent negative feedback mechanism to prevent excessive NMDAR activation and to maintain the receptor activity at the physiological level (Choi et al., 2000).

The mechanism underlying the NO modulation of NMDARs seems to involve a direct and cGMP-independent pathway. It has been found that NMDARs can be directly modified by a biochemical reaction, S-nitrosylation. S-nitrosylation is an important biological reaction of $\mathrm{NO}$ and is a key post-translational modification for multiple ion channels (Hess et al., 2005). Such modification adds a nitrosyl group $\left(\mathrm{NO}^{+}\right)$to the thiol side-chain of cysteine residues to form $S$-nitrosothiols, leading to a reversible change in tertiary structure and function of modified proteins (Sen and Snyder, 2010). In dissecting a molecular mechanism for the NO-NMDAR coupling, a single cysteine residue (C399) in the extracellular $\mathrm{N}$ terminus of NR2A was found to account for the predominant effect of NO. This cysteine can be physiologically S-nitrosylated after $\mathrm{NO}^{+}$transfer, and site-directed mutation of it to alanine abolished the NO-mediated inhibition of NR1/NR2A receptors (Choi et al., 2000). Thus, C399 S-nitrosylation serves as an important pathway linking NO to NMDARs. Additionally, two pairs of cysteine residues on NR1 (C744, C789) and NR2A (C87, C322) could undergo S-nitrosylation and thereby inhibit NMDARs if they are in the free thiol form (Choi et al., 2000, 2001; Lipton et al., 2002). Of note, nNOS forms a complex with NR1 via a scaffolding protein PSD-95 in the postsynaptic density microdomain (Brenman et al., 1996). Co-localization of these proteins at defined synaptic sites facilitates their direct interactions and enables rapid feedback inhibition of NMDARs via a NO-sensitive mechanism.

NO can also indirectly modulate NMDARs through Snitrosylating the prime regulators of NMDARs. Serine racemase (SR) is an enzyme which converts L-serine to D-serine, a co-agonist with glutamate at NMDARs. It has been shown that SR is physiologically S-nitrosylated for marked inhibition of the enzyme (Mustafa et al., 2007). This S-nitrosylation was enhanced by NMDAR-activated nNOS. Thus, postsynaptic stimulation of NO formation possibly feeds back to presynaptic cells to S-nitrosylate SR and decrease D-serine availability. This contributes to inhibiting excitatory synaptic transmission and preventing overstimulation of postsynaptic NMDARs. PSD-95 is a principal scaffolding protein at synaptic sites where it determines the number of NMDARs and thus efficiency and strength of the receptor. It also couples NMDARs to nNOS to form signalosomes for local NO production. Like SR, PSD-95 is physiologically S-nitrosylated (Ho et al., 2011). S-nitrosylation occurs at two specific cysteines (C3 and C5). Interestingly, these sites are also subject to palmitoylation, another reversible and cysteine-based post-translational modification which covalently attaches a fatty acid (palmitic acid) to cysteine (Ho et al., 2011). In cerebellar neurons, NO inhibited PSD-95 palmitoylation and decreased synaptic delivery of PSD95. In heterologous cells, decreased palmitoylation resulted in increased PSD-95 S-nitrosylation (Ho et al., 2011). These data support a model in which NMDARs stimulate NO to regulate 
PSD-95 via mutually competitive cysteine modifications. Regulated PSD-95 then reciprocally controls expression and activity of NMDARs.

Normal NMDAR activity is important for cell signaling and synaptic plasticity. Excessive activation of the receptor, however, results in excitotoxity and cell death. To this end, NMDARs are crucial for neuronal survival in various neurodegenerative disorders (Lipton and Rosenberg, 1994). Since NO inhibits excessive stimulation of NMDARs, it could consequently ameliorate NMDAR-mediated neurotoxicity. Of note, both NMDAR and NO activities are upregulated in parallel during neurological disorders such as ischemia, trauma, and inflammation. As such, they both are actively involved in resultant neuronal death under these neurodegenerative conditions (Dawson et al., 1993; Bonfoco et al., 1995). Indeed, hypoxia renders NMDARs exquisitely sensitive to NO-induced S-nitrosylation and inhibition of NMDARs in cortical neurons and oocytes (Takahashi et al., 2007). Such enhanced NO inhibition of NMDARs is thought to be neuroprotective and serves as a mechanism to counteract NMDAR-mediated cytotoxicity in hypoxia insults (Lei et al., 1992; Lipton et al., 1993; Choi et al., 2000; Takahashi et al., 2007). In addition, a large increase in NO levels in rat brains was discovered immediately after middle cerebral artery occlusion (Malinski et al., 1993). A higher level of NO, through the NO-mediated vasodilation, can increase cerebral blood flow and oxygen delivery (Toda et al., 2009). This, in concert with the NO inhibition of NMDARs, protects brain tissue during focal ischemia, although neuronal overproduction of NO may elicit neurotoxicity (Dalkara et al., 1994). The inhibitory control of NMDARs by NO has potential clinical implications. Pharmacotherapies targeting nitrosylatable cysteine residues can be developed to prevent NMDAR-mediated neurotoxicity during stroke (Takahashi et al., 2007).

\section{AMPARs}

AMPARs are another important glutamate ion channel. Like NMDARs, AMPARs become functional upon a heterotetrameric assembly of four subunits (GluR1-4 or GluRA-D; Dingledine et al., 1999). Most AMPARs contain symmetric "dimer of dimers" of GluR2 and either GluR1, GluR3, or GluR4 (Greger et al., 2007). Activation of these receptors promotes $\mathrm{Na}^{+}$influx and induces rapid depolarization. AMPARs open and close quickly and thus carry out the most of fast excitatory synaptic transmission in the CNS.

Little is known about whether AMPAR subunits are direct targets of NO. However, several key AMPAR-interacting proteins that actively control AMPAR trafficking, expression, and function are $\mathrm{S}$-nitrosylated and regulated by NO. $\mathrm{N}$-ethylmaleimide sensitive factor (NSF) is an ATPase enriched in neurons. It binds to GluR2 (Nishimune et al., 1998; Osten et al., 1998; Song et al., 1998), stabilizing or recycling AMPARs into postsynaptic membranes (Braithwaite et al., 2002; Lee et al., 2002). It was found that synaptic NSF was physiologically S-nitrosylated by endogenous, neuronally derived NO in mouse brain (Huang et al., 2005). NMDAR activation enhanced NSF S-nitrosylation and thereby augmented NSF binding to GluR2 and surface insertion of GluR2. These data reveal a NO-sensitive pathway linking NMDARs to AMPARs. Specifically, NMDARs, through activating associated nNOS, trigger NO formation, which upregulates NSF S-nitrosylation to promote its associations with GluR2 and increase surface expression of GluR2-containing AMPARs (Huang et al., 2005). In this process, PICK1 (protein interacting with $\mathrm{C}$ kinase 1), a synaptic anchoring protein binding and retaining GluR2 in intracellular pools, seems to mediate the NSF regulation of GluR2. S-nitrosylated NSF can dissemble the PICK1-GluR2 complex, leading to a release of GluR2 from PICK retention and subsequent surface delivery of GluR2/AMPARs (Hanley et al., 2002; Sossa et al., 2007).

Stargazin is another synaptic protein that links NO to AMPARs. As a founding member of the transmembrane AMPAR regulatory protein (TARP) family, stargazin serves as an auxiliary subunit of all AMPAR subtypes and directly interacts with a large part of AMPARs. As such, stargazin is thought to be a principal determinant of AMPAR surface expression. Indeed, Snyder and co-workers showed that stargazin, like NSF, is physiologically S-nitrosylated at C302 in primary neurons and heterologous cells (Selvakumar et al., 2009). S-nitrosylation of stargazin was positively linked to surface expression of this small tetraspanning membrane protein. S-nitrosylation also increased binding of stargazin to the AMPAR subunit GluR1, causing upregulated surface expression of the AMPAR. NMDAR stimulation increased S-nitrosylation of stargazin and binding of stargazin to AMPARs. Apparently, stargazin, like NSF, is a physiological regulator of AMPARs and a linker between NMDARs and AMPARs. Through a NO-sensitive mechanism, stargazin transmits NMDAR signals to the level of surface expression of AMPARs.

In addition to S-nitrosylation, NO stimulates soluble guanylyl cyclase to form the second messenger cGMP (Bredt and Snyder, 1989). Thus, cGMP and downstream protein kinases could constitute an indirect pathway to regulate AMPARs. A recent study supports this notion (Serulle et al., 2007). In this study, the cGMPdependent kinase type II (cGKII), a distinct GK isoform located in cellular membranes and broadly expressed in the brain (Francis and Corbin, 1999), was found to directly bind to GluR1. This binding was sensitive to nNOS activity and cGMP. Moreover, the binding enables the kinase to phosphorylate GluR1 at an intracellular serine site (S845). In cultured hippocampal neurons, active cGKII accumulated GluR1 on the plasma membrane at extrasynaptic sites. Blockade of cGKII prevented this accumulation and also an increase in mEPSC frequency and amplitude. Of note, $\mathrm{S} 845$ was also phosphorylated by protein kinase A (PKA), which was required for GluR1 synaptic insertion (Esteban et al., 2003). Thus, both cGKII and PKA pathways converge on S845 to regulate its phosphorylation and the phosphorylation-dependent GluR1 insertion into the surface membrane. It is believed that the two pathways operate in concert and in distinct temporospatial manners to modulate synaptic plasticity.

\section{MODULATION OF ASICs BY NO}

In addition to glutamate receptor channels, ASICs represent another family of ion channels subjected to the modulation by NO. Seven subunits of ASICs (1a, 1b1, 1b2, 2a, 2b, 3, and 4) have been identified to date, which are encoded by four genes (Price et al., 1996; Lingueglia et al., 1997; Waldmann et al., 1997a,b; Chen et al., 1998; Akopian et al., 2000). A major function of ASICs is to maintain normal cellular activity in response to changing $\mathrm{pH}$ 
signals. Given the fact that a variety of neurological disorders, including inflammation, ischemic stroke, traumatic brain injury and acid nociception, cause severe acidosis, ASICs are deemed to be activated and play a role in cell death and in the pathogenesis or progression of these diseases (Wemmie et al., 2006; Chu et al., 2011). As a result, ASICs are emerging as new pharmacotherapeutic targets for these illnesses (Wemmie et al., 2006; Xiong et al., 2008; Sluka et al., 2009; Xu and Duan, 2009).

Acid-sensing ion channels are subject to the regulation by various extracellular and intracellular signals (Chu et al., 2011). Among the known regulators is NO. It has been suggested that ASICs are a potential target for direct modulation by NOdependent S-nitrosylation (Cadiou et al., 2007). This is based on the facts that (1) the channel possesses a large number of cysteine residues on their extracellular domains (Waldmann et al., 1999), and (2) oxidizing agents, 5,5'-dithio-bis-(2-nitrobenzoic acid; DTNB), showed a sizable impact on acid-evoked currents in neurons and heterologous cells (Andrey et al., 2005; Chu et al., 2006). In support of this, Cadiou et al. (2007) reported that the NO donor SNAP potentiated proton-gated currents in rat cultured dorsal root ganglion (DRG) neurons and in CHO cells expressing each of ASIC subunits. The SNAP effect was not due to activation of the cGMP pathway because the cGMP analog db-cGMP did not mimic, and the guanylyl cyclase inhibitor ODQ did not block, the SNAP potentiation. The effect of SNAP was thought to be mediated via a direct mechanism involving NO S-nitrosylation at extracellular cysteines of ASICs (Cadiou et al., 2007).

Acid-sensing ion channels are known to participate in mediating acid-evoked pain in humans. This process is believed to be regulated by $\mathrm{NO}$, based on the finding that topical application of an NO donor enhanced acid-evoked pain (Cadiou et al., 2007). Another neurological disorder in which the NO potentiation of ASICs may have a pathophysiological relevance is ischemia. Cerebral ischemia is known to cause metabolic acidosis. This leads to activation of ASICs, especially $\mathrm{Ca}^{2+}$ permeable ASIC1a, which increases intracellular $\mathrm{Ca}^{2+}$ overload and results in neuronal death (Xiong et al., 2004). NO promoted such neuronal death by potentiating ASICs during mild and moderate acidosis, although in severe acidosis NO synthesis was inhibited and the NO contribution subsided (Jetti et al., 2010).

\section{CONCLUSION}

Ionotropic glutamate receptors have long been appreciated to be subject to various types of post-translational modifications which determine expression, trafficking, and function of modified

\section{REFERENCES}

Ahern, G. P., Klyachko, V. A., and Jackson, M. B. (2002). cGMP and Snitrosylation: two routes for modulation of neuronal excitability by NO. Trends Neurosci. 25, 510-517.

Akopian, A. N., Chen, C. C., Ding, Y., Cesare, P., and Wood, J. N. (2000). A new member of the acid-sensing ion channel family. Neuroreport 11, 2217-2222.
Alvarez de la Rosa, D., Krueger, S. R., Kolar, A., Shao, D., Fitzsimonds, Distribution, subcellular localization and ontogeny of ASIC1 in the mammalian central nervous system. J. Physiol. 546, 77-87.

Andrey, F., Tsintsadze, T., Volkova, T., Lozovaya, N., and Krishtal, O. (2005). Acid sensing ionic channels: modulation by redox reagents. R. M., and Canessa, C. M. (2003).

receptors. NO-dependent S-nitrosylation is one of these modifications. Available data show that NMDARs are directly modulated by NO-mediated S-nitrosylation. Direct S-nitrosylation occurs at a cysteine site (C399) in an extracellular N-terminal region of NR2A subunits. In addition to NR2A, NO influences other synaptic proteins that are important for NMDAR activity. Serine racemase which generates $\mathrm{D}$-serine, a co-agonist of NMDARs, is S-nitrosylated. The S-nitrosylation inhibits its enzymatic activity. PSD-95, a scaffolding protein stabilizing NMDARs at synaptic sites and linking nNOS to NMDARs, also undergoes physiological S-nitrosylation, which suppresses synaptic delivery of PSD-95. Apparently, the NO regulation of all three elements (NR2A, serine racemase, and PSD-95) can synergistically generate an inhibitory driving force on NMDARs. Given that NMDARs stimulate NO synthesis, NO forms a negative feedback loop to prevent overstimulation of the receptor.

Direct S-nitrosylation of AMPARs has not been reported. Instead, AMPAR-interacting proteins are S-nitrosylated and regulated by NO. NSF binds to AMPAR GluR2, while stargazin binds to GluR1. NMDAR activation enhances S-nitrosylation of both proteins, facilitating their binding to GluR1/2 and surface expression of AMPARs. In addition to this S-nitrosylation-dependent and cGMP-independent mechanism, the cGMP-dependent pathway also mediates the NO regulation of AMPARs. Like glutamate ion channels, ASICs are modulated by NO. NO is believed to potentiate ASIC activity. This potentiation was not mediated by the cGMP-dependent pathway, but mediated through S-nitrosylation of extracellular cysteines of ASICs. The NO potentiation of ASICs contributes to acid-evoked pain and ischemiainduced neuronal death. It is possible that all NO targets, including NMDARs, AMPARs, ASICs, and their associated proteins, can be co-localized at same subsets of synapses. NO could therefore simultaneously regulate these synaptic proteins and interactions among them to precisely control synaptic transmission. It is anticipated that NO modification biology of ion channels will grow rapidly and more sophisticated NO modification models on glutamate ion channels and ASICs will be characterized in vivo. Ultimately, this knowledge will be translated into novel and effective pharmacotherapies for respective neurological disorders.

\section{ACKNOWLEDGMENTS}

The work by the authors discussed in this article was supported by NIH R01 DA010355 and R01 MH061469 and a Saint Luke's grant (Saint Luke's Hospital Foundation, Kansas City).

Biochem. Biophys. Acta 1745, 1-6.

Bonfoco, E., Kraine, D., Ankarcrona, M., Nicotera, P., and Lipton, S. A. (1995). Apoptosis and necrosis: two distinct events induced respectively by mild and intense insults with NMDA or nitric oxide/superoxidein cortical cell cultures. Proc. Natl. Acad. Sci. U.S.A. 92, 7162-7166.

Braithwaite, S. P., Xia, H., and Malenka, R. C. (2002). Differential roles for
NSF and GRIP/ABP in AMPA receptor cycling. Proc. Natl. Acad. Sci. U.S.A. 99, 7096-7101.

Bredt, D. S., and Snyder, S. H. (1989). Nitric oxide mediates glutamatelinked enhancement of cGMP levels in the cerebellum. Proc. Natl. Acad. Sci. U.S.A. 86, 9030-9033.

Bredt, D. S., and Snyder, S. H. (1992). Nitric oxide, a novel neuronal messenger. Neuron 8, 3-11. 
Brenman, J. E., Chao, D. S., Gee, S. H., McGee, A. W., Graven, S. E., Santillano, D. R., Wu, Z., Huang, F., Xia, H., Peters, M. F., Froehner, S. C., and Bredt, D. S. (1996). Interaction of nitric oxide synthase with the postsynaptic density protein PSD-95 and alphal-syntrophin mediated by PDZ domains. Cell 84, 757-767.

Cadiou, H., Studer, M., Jones, N. G., Smith, E. S. J., Ballard, A., McMahon, S. B., and McNaughton, P. A. (2007). Modulation of acid-sensing ion channel activity by nitric oxide. J. Neurosci. 27, 13251-13260.

Chen, C. C., England, S., Akopian, A. N., and Wood, J. N. (1998). A sensory neuron-specific, proton-gated ion channel. Proc. Natl. Acad. Sci. U.S.A. 95, 10240-10245.

Choi, Y. B., Chen, H. V., and Lipton, S. A. (2001). Three pairs of cysteine residues mediate both redox and $\mathrm{Zn}^{2+}$ modulation of the NMDA receptor. J. Neurosci. 21, 392-400.

Choi, Y. B., Tenneti, L., Le, D. A., Ortiz, J., Bai, G., Chen, H. S. V., and Lipton, S. A. (2000). Molecular basis of NMDA receptor coupled ion channel modulation by S-nitrosylation. Nat. Neurosci. 3, 15-21.

Chu, X. P., Close, N., Saugstad, J. A., and Xiong, Z. G. (2006). ASIClaspecific modulation of acid-sensing ion channels in mouse cortical neurons by redox reagents. J. Neurosci. 26, 5329-5339.

Chu, X. P., Papasian, C. J., Wang, J. Q., and Xiong, Z. G. (2011). Modulation of acid-sensing ion channels: molecular mechanisms and therapeutic potential. Int. J. Physiol. Pathophysiol. Pharmacol. 3, 288-309.

Dalkara, T., Yoshida, T., Irikura, K., and Moskowitz, M. A. (1994). Dual role of nitric oxide in focal cerebral ischemia. Neuropharmacology 33, 1447-1452.

Dawson, V. L., Dawson, T. M., Bartley, D. A., Uhl, G. R., and Snyder, S. H. (1993). Mechanisms of nitric oxide-mediated neurotoxicity in primary brain cultures. J. Neurosci. 13, 2651-2661.

Dingledine, R. Borges, K., Bowie, D., and Traynelis, S. F. (1999). The glutamate receptor ion channel. Pharmacol. Rev. 51, 7-61.

Esteban, J. A., Shi, S. H., Wilson, C., Nuriya, M., Huganir, R. L., and Malinow, R. (2003). PKA phosphorylation of AMPA receptor subunits controls synaptic trafficking underlying plasticity. Nat. Neurosci. 6, 136-143.

Francis, S. H., and Corbin, J. D. (1999). Cyclic nucleotide-dependent protein kinases: intracellular receptors for cAMP and cGMP action. Crit. Rev. Clin. Lab. Sci. 36, 275-328.

Gao, J., Duan, B., Wang, D. G., Deng, X. H., Zhang, G. Y., Xu, L., and Xu, T. L. (2005). Coupling between NMDA receptor and acid-sensing ion channel contributes to ischemic neuronal death. Neuron 48, 635-646.

Garthwaite, J., Charles, S. L., and Chess, W. R. (1988). Endothelium-derived relaxing factor release on activation of NMDA receptors suggests role as intercellular messenger in the brain. Nature 336, 385-388.

Greger, I. H., Ziff, E. B., and Penn, A. C. (2007). Molecular determinants of AMPA receptor subunit assembly. Trends Neurosci. 30, 407-416.

Grifoni, S. C., Jernigan, N. L., Hamilton, G., and Drummond, H. A. (2008). ASIC proteins regulate smooth muscle cell migration. Microvasc. Res. 75, 202-210.

Hanley, J. G., Khatri, L., Hanson, P. I., and Ziff, E. B. (2002). NSF ATPase and alpha-/beta-SNAPs disassemble the AMPA receptor-PICK1 complex. Neuron 34, 53-67.

Hess, D. T., Matsumoto, A., Kim, S. O., Marshall, H. E., and Stamler, J. S. (2005). Protein S-nitrosylation: purview and parameters. Nat. Rev. Mol. Cell Biol. 6, 150-166.

Ho, G. P., Selvakumar, B., Mukai, J., Hester, L. D., Wang, Y., Gogos, J. A., and Snyder, S. H. (2011). Snitrosylation and S-palmitoylation reciprocally regulate synaptic targeting of PSD-95. Neuron 71, 131-141.

Huang, Y., Man, H. Y., Sekine-Aizawa, Y., Han, Y., Juluri, K., Luo, H., Cheah, J., Lowenstein, C., Huganir, R. L., and Snyder, S. H. (2005). Snitrosylation of N-ethylmaleimide sensitive factor mediates surface expression of AMPA receptors. Neuron 46, 533-540.

Jahr, H., van Driel, M., van Osch, G. J., Weinans, H., and van Leeuwen, J. P. (2005). Identification of acidsensing ion channels in bone. Biochem. Biophys. Res. Commun. 337, 349-354.

Jetti, S. K., Swain, S. M., Majumder, S., Chatterjee, S., Poornima, V., and Bera, A. K. (2010). Evaluation of the role of nitric oxide in acid sensing ion channel mediated cell death. Nitric Oxide 22, 213-219.

Kendrick, K. M., Guevara-Guzman, R., de la Riva, C., Christensen, J., Ostergaard, K., and Emson, P. C. (1996). NMDA and kainite-evoked release of nitric oxide and classical transmitters in the rat striatum: in vivo evidence that nitric oxide may play a neuroprotective role. Eur. J. Neurosci. 8, 2619-2634.
Krishtal, O. A., and Pidoplichko, V. I. (1980). A receptor for protons in the nerve cell membrane. Neuroscience 5, 2325-2327.

Lee, S. H., Liu, L., Wang, Y. T., and Sheng, M. (2002). Clathrin adaptor AP2 and NSF interact with overlapping sites of GluR2 and play distinct roles in AMPA receptor trafficking and hippocampal LTD. Neuron 36 , 661-674.

Lei, S. Z., Pan, Z. H., Aggarwal, S. K., Chen, H. S., Hartman, J., Sucher, N. J., and Lipton, S. A. (1992). Effect of nitric oxide production on the redox modulatory site of the NMDA receptor-channel complex. Neuron 8, 1087-1099.

Lingueglia, E., De Weille, J. R., Bassilana, F., Heurteaux, C., Sakai, H., Waldmann, R., and Lazdunski, M. (1997). A modulatory subunit of acid sensing ion channels in brain and dorsal root ganglion cells. J. Biol. Chem. 272, 29778-29783.

Lipton, S. A., Choi, Y. B., Pan, Z. H., Lei, S. Z., Chen, H. S., Sucher, N. J., Loscalzo, J., Singel, D. J., and Stamler, J. S. (1993). A redoxbased mechanism for the neuroprotective and neurodestructive effects of nitric oxide and related nitrosocompounds. Nature 364, 626-632.

Lipton, S. A., Choi, Y. B., Takahashi, T., Zhang, D., Li, W., Godzik, A., and Bankston, L. A. (2002). Cysteine regulation of protein function - as exemplified by NMDAreceptor modulation. Trends $\mathrm{Neu}$ rosci. 25, 474-480.

Lipton, S. A., Rayudu, P. V., Choi, Y. B., Sucher, N. J., and Chen, H. S. (1998). Redox modulation of the NMDA receptor by NO-related species. Prog. Brain Res. 118, 73-82.

Lipton, S. A., and Rosenberg, P. A. (1994). Mechanism of disease: excitatory amino acids as a final common pathway for neurologic disorders. N. Engl. J. Med. 330, 613-622.

Malinski, T., Bailey, F., Zhang, Z. G., and Chopp, M. (1993). Nitric oxide measured by a porphyrinic microsensor in rat brain after transient middle cerebral artery occlusion. J. Cereb. Blood Flow Metab. 13, 355-358.

Manzoni, O., and Bockaer, J. (1993). Nitric oxide synthase activity endogenously modulates NMDA receptors. J. Neurochem. 61 , 368-370.

Manzoni, O., Prezeau, L., Marin, P., Deshager, S., Bockaert, J., and Fagni, L. (1992). Nitric oxide-induced blockade of NMDA receptors. $\mathrm{Neu}$ ron 8, 653-662.

Mustafa, A. K., Kumar, M., Selvakumar, B., Ho, G. P., Ehmsen, J. T., Barrow,
R. K., Amzel, L. M., and Snyder, S. H. (2007). Nitric oxide S-nitrosylates serine racemase, mediating feedback inhibition of D-serine formation. Proc. Natl. Acad. Sci. U.S.A. 104, 2950-2955.

Nishimune, A., Isaac, J. T., Molnar, E., Noel, J., Nash, S. R., Tagaya, M., Collingridge, G. L., Nakanishi, S., and Henley, J. M. (1998). NSF binding to GluR2 regulates synaptic transmission. Neuron 21, 87-97.

Omerovic, A., Chen, S. J., Leonard, J. P., and Kelso, S. R. (1995). Subunit-specific redox modulation of NMDA receptors expressed in Xenopus oocytes. J. Recept. Signal Transduct. Res. 15, 811-827.

Osten, P., Srivastava, S., Inman, G. J., Vilim, F. S., Khatri, L., Lee, L. M., States, B. A., Einheber, S., Milner, T. A., Hanson, P. I., and Ziff, E. B. (1998). The AMPA receptor GluR2 C terminus can mediate a reversible, ATPdependent interaction with NSF and alpha- and beta-SNAPs. Neuron 21, 99-100.

Price, M. P., Snyder, P. M., and Welsh, M. J. (1996). Cloning and expression of a novel human brain $\mathrm{Na}^{+}$channel. J. Biol. Chem. 271, 7879-7882.

Selvakumar, B., Huganir, R. L., and Snyder, S. H. (2009). S-nitrosylation of stargazin regulates surface expression of AMPA-glutamate neurotransmitter receptors. Proc. Natl. Acad. Sci. U.S.A. 106, 16440-16445.

Sen, N., and Snyder, S. H. (2010). Protein modifications involved in neurotransmitter and gasotransmitter signaling. Trends Neurosci. 33, 493-502.

Serulle, Y., Zhang, S., Ninan, I., Puzzo, D., McCarthy, M., Khatri, L., Arancio, O., and Ziff, E. B. (2007). A GluR1-cGKII interaction regulates AMPA receptor trafficking. Neuron 56, 670-688.

Shibuki, K., and Okada, D. (1991). Endogenous nitric oxide release required for long-term synaptic depression in the cerebellum. Nature 349, 326-328.

Sluka, K. A., Winter, O. C., and Wemmie, J. A. (2009). Acid-sensing ion channels: a new target for pain and CNS diseases. Curr. Opin. Drug Discov. Devel. 12, 693-704.

Song, I., Kamboj, S., Xia, J., Dong, H., Liao, D., and Huganir, R. L. (1998). Interaction of the $\mathrm{N}$ ethylmaleimide-sensitive factor with AMPA receptors. Neuron 21, 393-400. 
Sossa, K. G., Beattie, J. B., and Carroll, R. C. (2007). AMPAR exocytosis through NO modulation of PICK1. Neuropharmacology 53, 92-100.

Stephenson, F. A. (2001). Subunit characterization of NMDA receptors. Curr. Drug Targets 2, 233-239.

Takahashi, H., Shin, Y., Cho, S. J., Zago, W. M., Nakamura, T., Gu, Z., Ma, Y., Furukawa, H., Liddington, R., Zhang, D., Tong, G., Chen, H. S., and Lipton, S. A. (2007). Hypoxia enhances S-nitrosylationmediated NMDA receptor inhibition via a thiol oxygen sensor motif. Neuron 53, 53-64.

Toda, N., Ayajiki, K., and Okamura, T. (2009). Cerebral blood flow regulation by nitric oxide: recent advances. Pharmacol. Rev. 61, 62-97.

Waldmann, R., Bassilana, F., de Weille, J., Champigny, G., Heurteaux, C., and Lazdunski, M. (1997a). Molecular cloning of a non-inactivating proton-gated $\mathrm{Na}^{+}$channel specific for sensory neurons. J. Biol. Chem. 272, 20975-20978.

Waldmann, R., Champigny, G., Bassilana, F., Heurteaux, C., and Lazdunski, M. (1997b). A proton-gated cation channel involved in acidsensing. Nature 386, 173-177.

Waldmann, R., Champigny, G., Lingueglia, E., de Weille, J. R. Heurteaux, C., and Lazdunski, M. (1999). H(+)-gated cation channels. Ann. N. Y. Acad. Sci. 868, 67-76.

Wemmie, J. A., Price, M. P., and Welsh, M. J. (2006). Acid-sensing ion channels: advances, questions and therapeutic opportunities. Trends $\mathrm{Neu}$ rosci. 29, 578-586.

Xiong, Z. G., Pignataro, G., Li, M., Chang, S. Y., and Simon, R. P. (2008). Acid-sensing ion channels (ASICs) as pharmacological targets for neurodegenerative diseases. Curr. Opin. Pharmacol. 8, 25-32.
Xiong, Z. G., Zhu, X. M., Chu, X. P., Minami, M., Hey, J., Wei, W. L., MacDonald, J. F., Wemmie, J. A., Price, M. P., Welsh, M. J., and Simon, R. P. (2004). Neuroprotection in ischemia: blocking calciumpermeable acid-sensing ion channels. Cell 118, 687-698.

Xu, T. L., and Duan, B. (2009). Calciumpermeable acid-sensing ion channel in nociceptive plasticity: a new target for pain control. Prog. Neurobiol. 87, 171-180.

Zha, X. M., Wemmie, J. A., Green, S. H., and Welsh, M. J. (2006). Acidsensing ion channel $1 \mathrm{a}$ is a postsynaptic proton receptor that affects the density of dendritic spines. Proc. Natl. Acad. Sci. U.S.A. 103, 16556-16561.

Conflict of Interest Statement: The authors declare that the research was conducted in the absence of any commercial or financial relationships that could be construed as a potential conflict of interest.

Received: 09 April 2012; paper pending published: 23 April 2012; accepted: 07 May 2012; published online: 24 May 2012.

Citation: Wang JQ, Chu X-P, Guo $M-L$, Jin $D-Z$, Xue B, Berry TJ, Fibuch EE and Mao L-M (2012) Modulation of ionotropic glutamate receptors and acid-sensing ion channels by nitric oxide. Front. Physio. 3:164. doi 10.3389/fphys.2012.00164

This article was submitted to Frontiers in Membrane Physiology and Biophysics, a specialty of Frontiers in Physiology. Copyright (C) 2012 Wang, Chu, Guo, Jin, Xue, Berry, Fibuch and Mao. This is an open-access article distributed under the terms of the Creative Commons Attribution Non Commercial License, which permits non-commercial use, distribution, and reproduction in other forums, provided the original authors and source are credited. 